\title{
Estrategias metodológicas para mejorar la enseñanza de contabilidad
}

\section{Methodological strategies to improve accounting education}

DOI: $10.46932 / \mathrm{sfjdv2n5-123}$

Received in: Oct 1st, 2021

Accepted in: Dec 30th, 2021

\author{
Liliana Isabel Flores Anchundia \\ Ingeniera en Administración Financiera. \\ Magister en Contabilidad y Auditoría.
}

Egresada en Maestría de Gestión Educativa. Docente en el Área de Contabilidad, Unidad Educativa José

María Velasco Ibarra, Buena Fe, Los Ríos, Ecuador.

calle arcadio fuente y transversal sin nombre junto al cementerio general.

E-mail: lili_isaldu@hotmail.es

Marjorie Magdalena Jara Jiménez

Ingeniera en Gestión Empresarial.

Magister en Administración de Empresas.

Docente de Emprendimiento, Unidad Educativa Mocache, Mocache, Los Ríos, Ecuador.

Dirección: Mocache km. 1 1 1/2 vía a Quevedo.

E-mail: clari_mar22@hotmail.com

Karen Lisseth Montúfar Chango

Ingeniera Química.

Magister en Psicología Educativa.

Docente en el Área de Ciencias Naturales, Unidad Educativa José María Velasco Ibarra, Buena Fe, Los Ríos, Ecuador.

Dirección: calle arcadio fuente y transversal sin nombre junto al cementerio general.

E-mail: karenmontufar@hotmail.com

\section{Teófilo Tonny Terán Jama}

Ingeniero en Contabilidad y auditoría.

Egresado en Maestría de Gestión Educativa, Docente en el Área de Contabilidad, Unidad Educativa José

María Velasco Ibarra, Buena Fe, Los Ríos, Ecuador.

calle arcadio fuente y transversal sin nombre junto al cementerio general.

E-mail: tonnyteran95@gmail.com

\section{RESUMEN}

La educación es uno de los elementos de mucha importancia para que los individuos se desarrollen en cualquier entorno social, en tal contexto, existe preocupación sobre la educación contable que gira en temáticas como la preparación del docente, currículos y modelos pedagógicos, por tal razón, este estudio tuvo como propósito determinar el efecto del diseño y aplicación de estrategias metodológicas para mejorar la enseñanza de contabilidad desde la percepción de los estudiantes. Se lo realizó bajo la metodología cuasiexperimental, con una muestra de 70 estudiantes distribuidos en grupos de 35 estudiantes uno experimental y otro de control. Para el recojo de la información se utilizó como instrumento un cuestionario el cual fue sometido a una validación de juicio de expertos y para garantizar su confiabilidad se realizó el análisis estadístico utilizando el coeficiente de Alfa de Cronbach. Como 
resultado se obtuvo que luego de la aplicación de las estrategias cambio el nivel de enseñanza de "mala" a "muy buena", obteniendo un valor "p" significativo de 0,000 según la U de Mann-Whitney y se concluyó que la aplicación de las estrategias metodológicas mejora de manera significativa la enseñanza de contabilidad según la percepción de los estudiantes.

Palabras Claves: Estrategias metodológicas, gamificación, Aprendizaje colaborativo, Aprendizaje basado en problemas, Enseñanza de contabilidad.

\begin{abstract}
Education is one of the very important elements for individuals to develop in any social environment, in such a context, there is concern about accounting education that revolves around topics such as teacher preparation, curricula and pedagogical models, for this reason, The purpose of this study was to determine the effect of the design and application of methodological strategies to improve the teaching of accounting from the students' perception. It was carried out under the quasi-experimental methodology, with a sample of 70 students distributed in groups of 35 students, one experimental and the other control. To collect the information, a questionnaire was used as an instrument, which was subjected to a validation of expert judgment and to guarantee its reliability, the statistical analysis was carried out using the Cronbach's Alpha coefficient. As a result, it was obtained that after the application of the strategies, the teaching level changed from "bad" to "very good", obtaining a significant "p" value of 0.000 according to the MannWhitney $\mathrm{U}$ and it is concluded that the application of The methodological strategies significantly improve the teaching of accounting according to the perception of the students.
\end{abstract}

Keywords: Methodological strategies, gamification, Collaborative learning, Problem-based learning, accounting teaching.

\title{
1 INTRODUCCIÓN
}

La educación es uno de los componentes que denotan mucha importancia para que los seres humanos puedan desarrollarse en cualquier entorno, especialmente en el entorno social y en el mundo actual, en tal contexto, las principales preocupaciones sobre la educación contable han girado en temáticas tales como la preparación del docente, currículo, modelos pedagógicos, lo que ha causado una construcción de discursos pedagógicos en algunos países Latinoamericanos y en algunos países europeos tales como España que coinciden en lineamientos y referencias para la construcción interdisciplinares tal cual se menciona en el trabajo de investigación de (Cuéllar, 2020)

En el entorno nacional, conforme a lo que determina el Ministerio de Educación (2016) en el ámbito de políticas educativas, emplear el uso de diversas actividades prácticas y el uso de herramientas tecnológicas facilita la enseñanza y aprendizaje del estudiante, de igual manera, en el área contable, convirtiéndose en la principal herramienta y la más esencial durante la enseñanza, ya que de esta manera se logra llegar al estudiante de modo que los contenidos les sean más fáciles de comprender, sin embargo, la educación en Ecuador se ha detenido en un sistema tradicionalista, conductista y arcaico, empleando métodos de enseñanzas contables pasivos y rutinarios, que en muchas ocasiones se tornan aburridos, lo 
cual causa en los estudiantes desmotivación, desinterés, apatía y que no permiten que la educación sea óptima y que satisfaga las exigencias de la nueva sociedad en que vivimos.

Las Instituciones Educativas Técnicas cuentan con docentes preparados y con la predisposición para enseñar a sus estudiantes en las áreas asignadas, sin embargo, el uso de estrategias metodológicas inadecuadas, crea dificultades educativas que afectan el normal desenvolvimiento de transformación y procedimiento de enseñanza aprendizaje, como se da el caso en la asignatura de contabilidad, esto puede deberse a que en ocasiones los docentes de contabilidad son formados en su profesión, más no en educación, por lo tanto, no cuentan con el conocimiento del uso de todas las herramientas didácticas y pedagógicas necesarias para lograr un mejor nivel de enseñanza, pues su enseñanza se enfoca en educar de manera memorista y transmitiendo de forma estática y mecánica la información contable, esta realidad crea barrera en los contenidos propuestos para la clase y los estudiantes se presentan más confusos para la comprensión de los mismos causando un deficiente desarrollo.

De acuerdo con las ideas que se han venido realizando, el objetivos general planteado en esta investigación es: Determinar el efecto del diseño y aplicación de estrategias metodológicas para mejorar la enseñanza de contabilidad desde la percepción de los estudiantes, por consiguiente, se plantea los siguientes objetivos específicos, primero, Identificar el nivel de uso de las estrategias metodológicas que utilizan los docentes para la enseñanza de contabilidad en las dimensiones de gamificación, aprendizaje colaborativo y aprendizaje basado en problemas, desde la percepción de los estudiantes de los grupos experimental y de control. En segundo lugar, Identificar los elementos que debe contener un programa de capacitación sobre estrategias metodológicas para la enseñanza de la contabilidad. Asimismo, es pertinente determinar la percepción que tienen los estudiantes del grupo experimental y de control, de la enseñanza de contabilidad que reciben de sus docentes, antes de la implementación de estrategias, y también establecer la percepción que tienen los estudiantes del grupo experimental y de control, de la enseñanza de contabilidad que reciben de sus docentes, después de la implementación de estrategias metodológicas, y por último, Evaluar los efectos que tiene la aplicación del programa de estrategias metodológicas según la percepción de los estudiantes de la enseñanza de la contabilidad, en cada una de sus dimensiones.

\section{METODOLOGÍA}

El presente estudio, se realizó desde el enfoque cuantitativo, como señala Gómez (2017), que éste enfoque, hace referencia a la recolección de datos, que es equivalente a medir, por otra parte, Galeano (2017), menciona que los estudios cuantitativos procuran de la explicación de una realidad social que se la observa de un punto de vista externo y objetivo con la finalidad de buscar la exactitud de mediciones, 
Alan \& Cortez (2018), a su vez manifiestan que la investigación cuantitativa está orientada a comprobar o verificar de manera deductiva las proposiciones planteadas.

Los métodos de investigación según la percepción de Rodríguez \& Pérez (2017), son las formas de organizar las actividades para alcanzar un objetivo, por lo que conceptualiza el análisis como un procedimiento que permite descomponer mentalmente un todo, la síntesis establece mentalmente la combinación de las partes analizadas y la deducción como un proceso que parte de lo general para llegar a afirmaciones particulares.

\section{RESULTADOS}

OE1. Identificar el nivel de uso de las estrategias metodológicas que utilizan los docentes para la enseñanza de contabilidad en las dimensiones de gamificación, aprendizaje colaborativo y aprendizaje basado en problemas, desde la percepción de los estudiantes de los grupos experimental y de control.

Tabla $N^{\circ}$ 03: Estrategias metodológicas que utilizan los docentes en la enseñanza de contabilidad.

\begin{tabular}{|c|c|c|c|c|c|c|c|c|c|c|}
\hline & \multicolumn{2}{|c|}{ Muy Bajo } & \multicolumn{2}{|c|}{ Bajo } & \multicolumn{2}{|c|}{ Medio } & \multicolumn{2}{|c|}{ Alto } & \multicolumn{2}{|c|}{ Total } \\
\hline & FI & $\%$ & FI & $\%$ & FI & $\%$ & FI & $\%$ & FI & $\%$ \\
\hline $\begin{array}{l}\text { Niveles de gamificación grupo } \\
\text { experimental }\end{array}$ & 6 & $17,1 \%$ & 18 & $51,4 \%$ & 9 & $25,7 \%$ & 2 & $5,7 \%$ & 35 & $100,0 \%$ \\
\hline $\begin{array}{l}\text { Niveles de gamificación en grupo de } \\
\text { control }\end{array}$ & 9 & $25,7 \%$ & 15 & $42,9 \%$ & 9 & $25,7 \%$ & 2 & $5,7 \%$ & 35 & $100,0 \%$ \\
\hline Niveles de AC grupo experimental & 16 & $45,7 \%$ & 12 & $34,3 \%$ & 5 & $14,3 \%$ & 2 & $5,7 \%$ & 35 & $100,0 \%$ \\
\hline Nivel de AC grupo de control & 14 & $40,0 \%$ & 15 & $42,9 \%$ & 4 & $11,4 \%$ & 2 & $5,7 \%$ & 35 & $100,0 \%$ \\
\hline Niveles de ABP grupo experimental & 21 & $60,0 \%$ & 12 & $34,3 \%$ & 1 & $2,9 \%$ & 1 & $2,9 \%$ & 35 & $100,0 \%$ \\
\hline Niveles del ABP de grupo de Control & 20 & $57,1 \%$ & 12 & $34,3 \%$ & 2 & $5,7 \%$ & 1 & $2,9 \%$ & 35 & $100,0 \%$ \\
\hline
\end{tabular}

Fuente: Cuestionario aplicado a estudiantes.

Como se observa en la tabla 03, el ABP obtuvo un porcentaje del 60,0\% en el GE y 57,1\% en el GC en nivel "Muy Bajo", seguido tenemos al AC con porcentajes de 45,7\% GE en un nivel "Muy bajo" y 42,9\% GC en nivel "Bajo", finalmente la gamificación la encontramos en porcentajes de 51,4\% GE y 42,9\% GC en nivel "Bajo". Es decir, que según la percepción de los estudiantes tanto del grupo experimental como en el grupo de control, si se utilizan las estrategias de gamificación, AC y ABP, pero en niveles bajos y muy bajos, siendo el ABP el menos utilizado por los docentes para la enseñanza de contabilidad.

OE2. Identificar los elementos que debe contener un programa de capacitación sobre estrategias metodológicas para la enseñanza de la contabilidad. 
Tabla No 04: Elementos de estrategias de Gamificación para la enseñanza de contabilidad.

\begin{tabular}{|c|c|c|c|c|c|c|c|c|c|c|}
\hline & \multicolumn{2}{|c|}{ Muy Bajo } & \multicolumn{2}{|c|}{ Bajo } & \multicolumn{2}{|c|}{ Medio } & \multicolumn{2}{|c|}{ Alto } & \multicolumn{2}{|c|}{ Total } \\
\hline & FI & $\%$ & FI & $\%$ & FI & $\%$ & FI & $\%$ & FI & $\%$ \\
\hline Incorpora el juego en el desarrollo de habilidades GE & 8 & $22,9 \%$ & 15 & $42,9 \%$ & 7 & $20,0 \%$ & 5 & $14,3 \%$ & 35 & $100,0 \%$ \\
\hline Incorpora el juego en el desarrollo de habilidades GC & 5 & $14,3 \%$ & 16 & $45,7 \%$ & 11 & $31,4 \%$ & 3 & $8,6 \%$ & 35 & $100,0 \%$ \\
\hline Hace uso del juego con herramientas virtuales GE & 10 & $28,6 \%$ & 23 & $65,7 \%$ & 2 & $5,7 \%$ & 0 & $0,0 \%$ & 35 & $100,0 \%$ \\
\hline Iso del juego con herramientas virtuales GC & 15 & $42,9 \%$ & 16 & $45,7 \%$ & 4 & $11,4 \%$ & 0 & $0,0 \%$ & 35 & $100,0 \%$ \\
\hline $\begin{array}{l}\text { Promueve la competencia en los juegos donde se } \\
\text { desarrollan habilidades para la contabilidad GE }\end{array}$ & 15 & $42,9 \%$ & 11 & $31,4 \%$ & 9 & $25,7 \%$ & 0 & $0,0 \%$ & 35 & $100,0 \%$ \\
\hline $\begin{array}{l}\text { Promueve la competencia en los juegos donde se } \\
\text { desarrollan habilidades para la contabilidad GC }\end{array}$ & 16 & $45,7 \%$ & 11 & $31,4 \%$ & 8 & $22,9 \%$ & 0 & $0,0 \%$ & 35 & $100,0 \%$ \\
\hline $\begin{array}{l}\text { Los logros alcanzados en los juegos tienen su } \\
\text { recompensa GE }\end{array}$ & 15 & $42,9 \%$ & 13 & $37,1 \%$ & 7 & $20,0 \%$ & 0 & $0,0 \%$ & 35 & $100,0 \%$ \\
\hline $\begin{array}{l}\text { Los logros alcanzados en los juegos tienen su } \\
\text { recompensa GC }\end{array}$ & 21 & $60,0 \%$ & 9 & $25,7 \%$ & 5 & $14,3 \%$ & 0 & $0,0 \%$ & 35 & $100,0 \%$ \\
\hline
\end{tabular}

Fuente: Cuestionario aplicado a estudiantes.

Como se observa en la tabla 04, existe similitud en los porcentajes de los indicadores que se han considerado para seleccionar los elementos que debe contener un programa de capacitación sobre estrategias metodológicas, tanto en el grupo experimental como en el grupo de control, encontrándose ambos grupos en los niveles "Bajo" y "Muy bajo", sin embargo, se destacaran los porcentajes más altos encontrados en el GE, obteniendo que en el indicador "Hace uso del juego con herramientas virtuales" de la estrategia metodológica de Gamificación, se obtuvo un 65,7\% en nivel "Bajo", mientras que los otros indicadores se los encontró dentro de un porcentaje del 42,9\% en niveles "Bajo" y "Muy Bajo". Esto quiere decir, que entre los elementos que debe contener un programa de capacitación sobre estrategias metodológicas para la enseñanza de la contabilidad se debió poner un mayor énfasis en los ítems que corresponden al indicador "Hace uso del juego con herramientas virtuales" al momento de su elaboración y aplicación.

Tabla No 05: Elementos de estrategias de Aprendizaje Colaborativo (AC) para la enseñanza de contabilidad.

\begin{tabular}{|c|c|c|c|c|c|c|c|c|c|c|}
\hline & \multicolumn{2}{|c|}{ Muy Bajo } & \multicolumn{2}{|r|}{ Bajo } & \multicolumn{2}{|c|}{ Medio } & \multicolumn{2}{|r|}{ Alto } & \multicolumn{2}{|r|}{ Total } \\
\hline & FI & $\%$ & FI & $\%$ & FI & $\%$ & FI & $\%$ & FI & $\%$ \\
\hline Fomenta el dialogo entre los estudiantes GE & 0 & $0,0 \%$ & 21 & $60,0 \%$ & 13 & $37,1 \%$ & 1 & $2,9 \%$ & 35 & $100,0 \%$ \\
\hline Fomenta el dialogo entre los estudiantes GC & 0 & $0,0 \%$ & 19 & $54,3 \%$ & 15 & $42,9 \%$ & 1 & $2,9 \%$ & 35 & $100,0 \%$ \\
\hline $\begin{array}{l}\text { Incentiva a practicar estrategias de resolución de } \\
\text { problemas GE }\end{array}$ & 0 & $0,0 \%$ & 21 & $60,0 \%$ & 10 & $28,6 \%$ & 4 & $11,4 \%$ & 35 & $100,0 \%$ \\
\hline $\begin{array}{l}\text { Incentiva a practicar estrategias de resolución de } \\
\text { problemas GC }\end{array}$ & 0 & $0,0 \%$ & 18 & $51,4 \%$ & 14 & $40,0 \%$ & 3 & $8,6 \%$ & 35 & $100,0 \%$ \\
\hline $\begin{array}{l}\text { Promueve a que los estudiantes se enseñen } \\
\text { mutuamente a dominar temáticas contables y } \\
\text { desarrollen competencias GE }\end{array}$ & 0 & $0,0 \%$ & 25 & $71,4 \%$ & 9 & $25,7 \%$ & 1 & $2,9 \%$ & 35 & $100,0 \%$ \\
\hline $\begin{array}{l}\text { Promueve a que los estudiantes se enseñen } \\
\text { mutuamente a dominar temáticas contables y } \\
\text { desarrollen competencias GC }\end{array}$ & 0 & $0,0 \%$ & 27 & $77,1 \%$ & 6 & $17,1 \%$ & 2 & $5,7 \%$ & 35 & $100,0 \%$ \\
\hline $\begin{array}{l}\text { Hace uso de la redacción para enseñar contenidos } \\
\text { y competencias importantes GE }\end{array}$ & 0 & $0,0 \%$ & 24 & $68,6 \%$ & 10 & $28,6 \%$ & 1 & $2,9 \%$ & 35 & $100,0 \%$ \\
\hline $\begin{array}{l}\text { Hace uso de la redacción para enseñar contenidos } \\
\text { y competencias importantes GC }\end{array}$ & 0 & $0,0 \%$ & 25 & $71,4 \%$ & 8 & $22,9 \%$ & 2 & $5,7 \%$ & 35 & $100,0 \%$ \\
\hline
\end{tabular}

Fuente: Cuestionario aplicado a estudiantes. 
Como se observa en la tabla 05, existe similitud en los porcentajes de los indicadores que se han considerado para seleccionar los elementos que debe contener un programa de capacitación sobre estrategias metodológicas, tanto en el GE como en el GC, encontrándose ambos grupos en el nivel "Bajo", sin embargo, se destacaran los porcentajes más altos encontrados en el GE, obteniendo que en el indicador "Promueve a que los estudiantes se enseñen mutuamente a dominar temáticas contables y desarrollen competencias" de la estrategia metodológica AC, se obtuvo un 71,4\% en nivel "Bajo", mientras que el indicador "Hace uso de la redacción para enseñar contenidos y competencias importantes de la contabilidad" obtuvo un porcentaje de 68,6\% en nivel "Bajo". Es decir, que entre los elementos que debe contener un programa de capacitación sobre estrategias metodológicas para la enseñanza de la contabilidad en el AC, se debió poner un mayor énfasis en los ítems que corresponden a los indicadores "Promueve a que los estudiantes se enseñen mutuamente a dominar temáticas contables y desarrollen competencias" y "Hace uso de la redacción para enseñar contenidos y competencias importantes de la contabilidad" al momento de su elaboración y aplicación.

Tabla No 06: Elementos de estrategias de Aprendizaje Basado en Problemas (ABP) para la enseñanza de contabilidad.

\begin{tabular}{|c|c|c|c|c|c|c|c|c|c|c|}
\hline & \multicolumn{2}{|c|}{ Muy Bajo } & \multicolumn{2}{|c|}{ Bajo } & \multicolumn{2}{|c|}{ Medio } & \multicolumn{2}{|c|}{ Alto } & \multicolumn{2}{|c|}{ Total } \\
\hline & FI & $\%$ & FI & $\%$ & FI & $\%$ & FI & $\%$ & FI & $\%$ \\
\hline $\begin{array}{l}\text { Se motiva a los estudiantes presentando } \\
\text { problemas reales GE }\end{array}$ & 20 & $57,1 \%$ & 12 & $34,3 \%$ & 3 & $8,6 \%$ & 0 & $0,0 \%$ & 35 & $100,0 \%$ \\
\hline $\begin{array}{l}\text { Se motiva a los estudiantes presentando } \\
\text { problemas reales GC }\end{array}$ & 24 & $68,6 \%$ & 8 & $22,9 \%$ & 3 & $8,6 \%$ & 0 & $0,0 \%$ & 35 & $100,0 \%$ \\
\hline $\begin{array}{l}\text { Se promueve el trabajo en equipo para resolver } \\
\text { problemas contables GE }\end{array}$ & 23 & $65,7 \%$ & 11 & $31,4 \%$ & 1 & $2,9 \%$ & 0 & $0,0 \%$ & 35 & $100,0 \%$ \\
\hline $\begin{array}{l}\text { Se promueve el trabajo en equipo para resolver } \\
\text { problemas contables GC }\end{array}$ & 23 & $65,7 \%$ & 9 & $25,7 \%$ & 3 & $8,6 \%$ & 0 & $0,0 \%$ & 35 & $100,0 \%$ \\
\hline $\begin{array}{l}\text { El docente interactúa como un tutor para la } \\
\text { resolución de problemas GE }\end{array}$ & 25 & $71,4 \%$ & 8 & $22,9 \%$ & 2 & $5,7 \%$ & 0 & $0,0 \%$ & 35 & $100,0 \%$ \\
\hline $\begin{array}{l}\text { El docente interactúa como un tutor para la } \\
\text { resolución de problemas GC }\end{array}$ & 24 & $68,6 \%$ & 9 & $25,7 \%$ & 2 & $5,7 \%$ & 0 & $0,0 \%$ & 35 & $100,0 \%$ \\
\hline $\begin{array}{l}\text { Se impulsa a la participación activa para la } \\
\text { identificación, investigación y resolución de } \\
\text { problemas contables GE }\end{array}$ & 12 & $34,3 \%$ & 20 & $57,1 \%$ & 2 & $5,7 \%$ & 1 & $2,9 \%$ & 35 & $100,0 \%$ \\
\hline $\begin{array}{l}\text { Se impulsa a la participación activa para la } \\
\text { identificación, investigación y resolución de } \\
\text { problemas contables GC }\end{array}$ & 11 & $31,4 \%$ & 18 & $51,4 \%$ & 5 & $14,3 \%$ & 1 & $2,9 \%$ & 35 & $100,0 \%$ \\
\hline
\end{tabular}

Fuente: Cuestionario aplicado a estudiantes

Como se observa en la tabla 06, existe similitud en los porcentajes de los indicadores que se han considerado para seleccionar los elementos que debe contener un programa de capacitación sobre estrategias metodológicas, tanto en el GE como en el GC, hallándose ambos grupos en el niveles "Muy bajo" y "Bajo", sin embargo, se destacaran los porcentajes más altos encontrados en GE, obteniéndose en el indicador "El docente interactúa como un tutor para la resolución de problemas" de la estrategia metodología ABP, que obtuvo un 71,4\% en nivel "Muy Bajo", mientras que el indicador "Se promueve 
el trabajo en equipo para resolver problemas contables" obtuvo un porcentaje de 65,7\% en nivel "Muy Bajo", los demás indicadores se los encontró dentro de un porcentaje del 57,1\% en el niveles "Muy Bajo" y "Bajo". Es decir, que entre los elementos que debe contener un programa de capacitación sobre estrategias metodológicas para la enseñanza de la contabilidad en el ABP, se debió poner un mayor énfasis en los ítems que corresponden a los indicadores "El docente interactúa como un tutor para la resolución de problemas" y "Se promueve el trabajo en equipo para resolver problemas contables" al momento de su elaboración y aplicación.

OE3. Determinar la percepción que tienen los estudiantes del grupo experimental y de control, de la enseñanza de contabilidad que reciben de sus docentes, antes de la implementación de estrategias.

Tabla $N^{\circ}$ 07: Percepción que tienen los estudiantes del grupo experimental y de control, de la enseñanza de contabilidad que reciben de sus docentes antes (pre test) de la implementación de estrategias.

\begin{tabular}{lrrrr}
\hline & \multicolumn{2}{c}{ Grupo Experimental } & \multicolumn{2}{c}{ Grupo Control } \\
\cline { 2 - 5 } & FI & \multicolumn{1}{c}{ FI } & 20 & $\%$ \\
\hline Mala & 21 & $60,0 \%$ & 13 & $57,1 \%$ \\
Regular & 12 & $34,3 \%$ & 1 & $37,1 \%$ \\
Buena & 1 & $2,9 \%$ & 1 & $2,9 \%$ \\
Muy Buena & 1 & $2,9 \%$ & 35 & $2,9 \%$ \\
Total & 35 & $100,0 \%$ & $100,0 \%$ \\
\hline
\end{tabular}

Fuente: Cuestionario aplicado a estudiantes.

Como se observa en la tabla 07, la percepción que tienen los estudiantes del GE y del GC, de la enseñanza de contabilidad que reciben de sus docentes, se encuentran los porcentajes más altos en los niveles "Mala" y "Regular", teniendo en el GE un 60\% y el GC un 57,1\% en nivel "Mala" y en el nivel "Regular" se observa el 34,3\% en el GE y el 37,1\% en el GC. Esto quiere decir, que en ambos grupos no se tiene una buena percepción de la enseñanza de contabilidad que reciben de sus docentes, por lo que es pertinente la aplicación de nuevas estrategias para tratar de mejorarla.

\subsection{CONTRASTACIÓN DE HIPÓTESIS}

Antes de iniciar con la contrastación de hipótesis, es necesario someter los datos la prueba de normalidad, que permita determinar el tipo de estadístico a utilizar (paramétrico o no paramétrico), para la prueba de hipótesis.

Tabla $N^{\circ} 08$ Prueba de normalidad

\begin{tabular}{lccc}
\hline & \multicolumn{3}{c}{ Shapiro-Wilk } \\
\cline { 2 - 4 } & Estadístico & gl & Sig. \\
\hline Pret_Gexp & 0,913 & 35 & 0,009 \\
Pret_Gcont & 0,886 & 35 & 0,002 \\
\hline
\end{tabular}

Los resultados obtenidos en la tabla 08, muestran que los valores de significancia del grupo experimental y de control se encuentran por debajo del 0,05 por lo que según el autor Shapiro-Wilk, estos 
datos demuestran que no son normales y se sugiere utilizar pruebas no paramétricas para contrastación de las hipótesis.

\subsection{CONTRASTACIÓN DE LA HIPÓTESIS ESPECÍFICA 1}

$\mathbf{H}_{\mathrm{i}} \mathbf{1}$ : Antes de aplicar el programa con estrategias metodológicas, la percepción de la enseñanza que reciben en contabilidad de los estudiantes del grupo experimental y de control son similares.

Ho1: Antes de aplicar el programa con estrategias metodológicas, la percepción de la enseñanza que reciben en contabilidad de los estudiantes del grupo experimental y de control no son similares.

Prueba U de Mann-Whitney

Tabla $\mathrm{N}^{\circ} 09$ Rangos pre test y estadístico de prueba, grupo de experimental y de control.

\begin{tabular}{cccccc}
\hline \multirow{2}{*}{ Test } & Grupos & $\mathrm{N}$ & $\begin{array}{c}\text { Rango } \\
\text { promedio }\end{array}$ & $\begin{array}{c}\text { U de Mann- } \\
\text { Whitney }\end{array}$ & $\begin{array}{c}\text { Sig. } \\
\text { asintótica(bilateral) }\end{array}$ \\
\hline \multirow{3}{*}{ Pre test } & Experimental & 35 & 34,47 & \multirow{2}{*}{576,500} &, 672 \\
& Control & 35 & 36,53 & & \\
& Total & 70 & & & \\
\hline
\end{tabular}

Como se puede observar en la tabla 09, la prueba de U de Mann-Whitney fue de 576,500 y el valor significativo (bilateral) de 0,672 , lo que refiere que existe homogeneidad entre ambos grupos con respecto a la opinión que tienen los estudiantes, sobre la enseñanza de contabilidad que reciben de sus docentes, concluyendo así, que antes de la aplicación del programa con estrategias metodológicas, los estudiantes del grupo experimental y de control tienen una percepción de la enseñanza que reciben en contabilidad similar, por lo tanto se decide aceptar la hipótesis de investigación y se rechaza la nula.

OE4. Establecer la percepción que tienen los estudiantes del grupo experimental y de control, de la enseñanza de contabilidad que reciben de sus docentes, después de la implementación de estrategias metodológicas.

Tabla $N^{\circ}$ 10: Percepción que tienen los estudiantes del grupo experimental y de control, de la enseñanza de contabilidad que reciben de sus docentes, después (post test) de la implementación de estrategias metodológicas.

\begin{tabular}{lrrrr}
\hline & \multicolumn{2}{c}{ Grupo Experimental } & Grupo Control \\
\cline { 2 - 6 } & FI & $\%$ & & $\%$ \\
\hline Mala & 0 & $0,0 \%$ & 22 & $62,9 \%$ \\
Regular & 0 & $0,0 \%$ & 12 & $34,3 \%$ \\
Buena & 4 & $11,4 \%$ & 0 & $0,0 \%$ \\
Muy Buena & 31 & $88,6 \%$ & 1 & $2,9 \%$ \\
Total & 35 & $100,0 \%$ & 35 & $100,0 \%$ \\
\hline
\end{tabular}

Fuente: Cuestionario aplicado a estudiantes.

Como se observa en la tabla 10, sobre la percepción que tienen los estudiantes del GE y GC de la enseñanza de contabilidad que reciben de sus docentes, después de la implementación de estrategias metodológicas, encontramos que el GE cuenta con un porcentaje del 88,6\% en nivel "muy bueno" 
mientras que el GC obtiene un 62,9\% en nivel "mala". Lo que quiere decir, que la aplicación de las estrategias metodológicas en el grupo experimental influyó, generando diferencias con el grupo de control.

\subsection{CONTRASTACIÓN DE LA HIPÓTESIS ESPECÍFICA 2}

Hi2: Después de aplicar el programa con estrategias metodológicas, existe diferencia significativa de la percepción que tienen los estudiantes del grupo experimental y de control sobre la enseñanza que reciben en contabilidad.

H02: Después de aplicar el programa con estrategias metodológicas, no existe diferencia significativa de la percepción que tienen los estudiantes del grupo experimental y de control sobre la enseñanza que reciben en contabilidad.

Prueba U de Mann-Whitney

Tabla $N^{\circ} 11$ Rangos pos test y estadístico de prueba, grupo de experimental y de control

\begin{tabular}{lllccc}
\hline \multirow{2}{*}{ Test } & Grupos & $\mathrm{N}$ & $\begin{array}{c}\text { Rango } \\
\text { promedio }\end{array}$ & $\begin{array}{c}\text { U de Mann- } \\
\text { Whitney }\end{array}$ & $\begin{array}{c}\text { Sig. } \\
\text { asintótica(bilateral) }\end{array}$ \\
\hline \multirow{3}{*}{ Post test } & Experimental & 35 & 52,57 & \multirow{2}{*}{15,000} & \multirow{2}{*}{000} \\
& Control & 35 & 18,43 & & \\
& Total & 70 & & & \\
\hline
\end{tabular}

Como se puede observar en la tabla 11, la prueba de U de Mann-Whitney fue de 15,000 y el valor significativo (bilateral) de 0,00 , lo que refiere que existe disimilitud entre ambos grupos con respecto a la enseñanza de contabilidad que reciben los estudiantes de sus docentes después de la implementación de estrategias, concluyendo así, que con la aplicación del programa con estrategias metodológicas, los estudiantes del grupo experimental y de control tienen una percepción con diferencias significativas, por lo tanto, se decide aceptar la hipótesis de investigación y se rechaza la nula.

OE5. Evaluar los efectos que tiene la aplicación del programa de estrategias metodológicas según la percepción de los estudiantes de la enseñanza de la contabilidad, en cada una de sus dimensiones.

Tabla $\mathrm{N}^{\circ} 12$ Efectos de la aplicación de la estrategia metodológica de gamificación.

\begin{tabular}{lcccc}
\hline & \multicolumn{4}{c}{ Gamificación Grupo Experimental } \\
\cline { 2 - 5 } & \multicolumn{3}{c}{ Pre test } & Post test \\
\cline { 2 - 5 } & FI & $\%$ & 0 & $\%$ \\
\hline Muy Bajo & 6 & $17,1 \%$ & 0 & $0,0 \%$ \\
Bajo & 18 & $51,4 \%$ & 5 & $0,0 \%$ \\
Medio & 9 & $25,7 \%$ & 30 & $14,3 \%$ \\
Alto & 2 & $5,7 \%$ & $85,7 \%$ \\
Total & 35 & $100,0 \%$ & 35 & $100,0 \%$ \\
\hline
\end{tabular}

Fuente: Cuestionario aplicado a estudiantes.

Como se puede observar en la tabla 12, sobre los efectos de la aplicación de la estrategia metodológica de gamificación en el GE el porcentaje más alto obtenido en el pre test se lo encontraba en 
el nivel "Bajo" con un 51,4\%, en el pos test el porcentaje más alto se lo encuentra en el nivel "Alto" con el $85,7 \%$. Esto quiere decir, que después de la aplicación del programa de estrategias metodológicas se obtuvo un efecto positivo en los estudiantes.

Tabla $\mathrm{N}^{\circ} 13$ Efectos de la aplicación de la estrategia metodológica de aprendizaje colaborativo.

\begin{tabular}{lcccc}
\hline & \multicolumn{3}{c}{ Aprendizaje Colaborativo Grupo Experimental } \\
\cline { 2 - 5 } & \multicolumn{2}{c}{ Pre test } & FI & Post test \\
\cline { 2 - 5 } & FI & $45,7 \%$ & 0 & $0,0 \%$ \\
\hline Muy Bajo & 16 & $34,3 \%$ & 0 & $0,0 \%$ \\
Bajo & 12 & $14,3 \%$ & 7 & $20,0 \%$ \\
Medio & 5 & $5,7 \%$ & 28 & $80,0 \%$ \\
Alto & 2 & $100,0 \%$ & 35 & $100,0 \%$ \\
Total & 35 & &
\end{tabular}

Fuente: Cuestionario aplicado a estudiantes

Como se puede observar en la tabla 13, sobre los efectos de la aplicación de la estrategia metodológica de aprendizaje colaborativo en el GE el porcentaje más alto obtenido en el pre test se lo encontraba en el nivel "Muy bajo" con un 45,7\%, en el pos test el porcentaje más alto se lo encuentra en el nivel "Alto" con el 80,0\%. Esto quiere decir, que después de la aplicación del programa de estrategias metodológicas se obtuvo un efecto positivo en los estudiantes.

Tabla $\mathrm{N}^{\circ} 14$ Efectos de la aplicación de la estrategia metodológica de aprendizaje basado en problemas.

\begin{tabular}{lcccc}
\hline & \multicolumn{3}{c}{ Aprendizaje Basado en Problemas Grupo Experimental } \\
\cline { 2 - 5 } & \multicolumn{2}{c}{ Pre test } & FI & Post test \\
\cline { 2 - 5 } & FI & $\%$ & 0 & $0,0 \%$ \\
\hline Muy Bajo & 21 & $60,0 \%$ & 1 & $2,9 \%$ \\
Bajo & 12 & $34,3 \%$ & 23 & $65,7 \%$ \\
Medio & 1 & $2,9 \%$ & 11 & $31,4 \%$ \\
Alto & 1 & $2,9 \%$ & 35 & $100,0 \%$ \\
Total & 35 & $100,0 \%$ & \\
\hline
\end{tabular}

Fuente: Cuestionario aplicado a estudiantes

Como se puede observar en la tabla 14, sobre los efectos de la aplicación de la estrategia metodológica de aprendizaje basado en problemas en el GE el porcentaje más alto obtenido en el pre test se lo encontraba en el nivel "Muy bajo" con un $60,0 \%$, en el pos test el porcentaje más alto se lo encuentra en el nivel "Medio" con el 65,7\%. Esto quiere decir, que después de la aplicación del programa de estrategias metodológicas se obtuvo un efecto positivo en los estudiantes, sin embargo, los resultados obtenidos demuestran que pese a que se aplicó la estrategia metodológica ABP de igual manera que las otras estrategias, esta no causo mayor impacto en los estudiantes, pues se mantiene en un nivel "medio" por lo que se asume que no es una estrategia apropiada para la enseñanza de asignatura de contabilidad. 


\subsection{CONTRASTACIÓN DE LA HIPÓTESIS ESPECÍFICA 3}

$\mathbf{H}_{\mathbf{i}} 3$ : La aplicación del programa con cada una de las estrategias metodológicas utilizadas, tuvo un efecto positivo en la percepción de los estudiantes del grupo experimental sobre la enseñanza de contabilidad.

H03: La aplicación del programa con cada una de las estrategias metodológicas utilizadas, no tuvo un efecto positivo en la percepción de los estudiantes del grupo experimental sobre la enseñanza de contabilidad.

Prueba U de Mann-Whitney

Tabla $\mathrm{N}^{\circ} 15$ Rangos pre test y post test y estadístico de prueba, grupo de experimental

\begin{tabular}{lllccc}
\hline & Test & $\mathrm{N}$ & $\begin{array}{c}\text { Rango } \\
\text { promedio }\end{array}$ & $\begin{array}{c}\text { U de Mann- } \\
\text { Whitney }\end{array}$ & $\begin{array}{c}\text { Sig. } \\
\text { asintótica(bilateral) }\end{array}$ \\
\hline \multirow{3}{*}{ Gamificación } & Pre test & 35 & 19,23 & 43,000 & 0,000 \\
& Post test & 35 & 51,77 & & \\
& Total & 70 & & & \multirow{2}{*}{0,000} \\
& Pre test & 35 & 18,89 & \multirow{2}{*}{31,000} & \\
Aprendizaje colaborativo & Post test & 35 & 52,11 & & \multirow{2}{*}{0,000} \\
& Total & 70 & & & \\
\hline \multirow{2}{*}{ Aprendizaje basado en } & Pre test & 35 & 19,74 & \multirow{2}{*}{61,000} & \\
problemas & Post test & 35 & 51,26 & & \\
& Total & 70 & & & \\
& Fuente: Cuestionario aplicado a estudiantes.
\end{tabular}

Como se puede observar en la tabla 15 la prueba de U de Mann-Whitney fue de 43,000 en la gamificación, 31,000 en el aprendizaje colaborativo y el 61,000 en el aprendizaje basado en problemas, mientras que el valor significativo (bilateral) de las tres dimensiones es de 0,000 , lo que refiere que existe disimilitud entre el pre test y pos test, con respecto a los efectos en la percepción de los estudiantes del grupo experimental sobre la enseñanza de contabilidad, concluyendo así, que con la aplicación del programa con estrategias metodológicas, existió diferencia significativa, por lo tanto, se decide aceptar la hipótesis de investigación y rechazar la nula.

OG. Determinar el efecto del diseño y aplicación de estrategias metodológicas para mejorar la enseñanza de contabilidad desde la percepción de los estudiantes. 
Tabla $N^{\circ} 16$ Efectos del diseño y aplicación de estrategias metodológicas para mejorar la enseñanza de contabilidad desde la percepción de los estudiantes.

\begin{tabular}{|c|c|c|c|c|}
\hline & \multicolumn{4}{|c|}{ Percepción de enseñanza de contabilidad Grupo Experimental } \\
\hline & \multicolumn{2}{|c|}{ Pre test } & \multicolumn{2}{|c|}{ Post test } \\
\hline & FI & $\%$ & FI & $\%$ \\
\hline Mala & 21 & $60,00 \%$ & 0 & $0,00 \%$ \\
\hline Regular & 12 & $34,30 \%$ & 0 & $0,00 \%$ \\
\hline Buena & 1 & $2,90 \%$ & 4 & $11,40 \%$ \\
\hline Muy Buena & 1 & $2,90 \%$ & 31 & $88,60 \%$ \\
\hline Total & 35 & $100,00 \%$ & 35 & $100,00 \%$ \\
\hline
\end{tabular}

Fuente: Cuestionario aplicado a estudiantes.

Como se observar en la tabla 16, sobre los efectos del diseño y aplicación de estrategias metodológicas para mejorar la enseñanza de contabilidad desde la percepción de los estudiantes en el GE el porcentaje más alto obtenido en el pre test se lo encontraba en el nivel "Mala" con un 60,0\%, en el pos test el porcentaje más alto se lo encuentra en el nivel "Muy Buena" con el 88,6\%. Esto quiere decir, que después de la aplicación del programa de estrategias metodológicas se obtuvo un efecto favorable en los estudiantes, mejorando de manera significativa la enseñanza de contabilidad desde la percepción de los estudiantes.

\subsection{CONTRASTACIÓN DE LA HIPÓTESIS GENERAL}

$\mathbf{H}_{\mathbf{i}} \mathbf{G}$ : El diseño y aplicación de estrategias metodológicas mejora de manera significativa la enseñanza de contabilidad según la percepción de los estudiantes.

HoG: El diseño y aplicación de estrategias metodológicas no mejora de manera significativa la enseñanza de contabilidad según la percepción de los estudiantes.

Prueba U de Mann-Whitney

Tabla $N^{\circ} 17$ Rangos pre test y post test y estadístico de prueba, grupo de experimental.

\begin{tabular}{lllccc}
\hline & Test & $\mathrm{N}$ & $\begin{array}{c}\text { Rango } \\
\text { promedio }\end{array}$ & $\begin{array}{c}\text { U de Mann- } \\
\text { Whitney }\end{array}$ & $\begin{array}{c}\text { Sig. } \\
\text { asintótica(bilateral) }\end{array}$ \\
\hline \multirow{3}{*}{ Grupo } & Pre test & 35 & 18,13 & \multirow{2}{*}{, 000} \\
Experimental & Post test & 35 & 52,87 & 4,500 & \\
& Total & 70 & & & \\
& & & & \\
\hline
\end{tabular}

Fuente: Cuestionario aplicado a estudiantes.

Como se puede observar en la tabla 17 la prueba de U de Mann-Whitney fue de 4,500 y el valor significativo (bilateral) de 0,000, lo que refiere que existe disimilitud entre el pre test y pos test, con respecto a los efectos de la aplicación del programa de estrategias metodológicas en la percepción de los estudiantes sobre la enseñanza de contabilidad, concluyendo así, que con la aplicación del programa con estrategias metodológicas, existió diferencia significativa, por lo tanto, se decide aceptar la hipótesis de investigación y rechazar la nula. 


\section{DISCUSIÓN}

El proceso de discusión de los resultados en la presente investigación titulada estrategias metodológicas para mejorar la enseñanza de contabilidad en las Instituciones Educativas Técnicas, la misma se ha estructurado por objetivos de acuerdo a los lineamientos institucionales, por lo tanto, en el primer objetivo específico, Identificar el nivel de uso de las estrategias metodológicas que utilizan los docentes para la enseñanza de contabilidad en las dimensiones de gamificación, aprendizaje colaborativo y aprendizaje basado en problemas, desde la percepción de los estudiantes de los grupos experimental y de control.

Se encontró, que luego de haber realizado el pre test tanto en el grupo experimental como en el de control se obtuvo que según la percepción de los estudiantes en ambos grupos si se aplican las estrategias antes mencionadas pero en niveles "muy bajo" y "bajo" siendo el aprendizaje basado en problemas el menos aplicado con un porcentaje del 60,0\% en el grupo experimental y 57,1\% en el grupo de control con nivel "Muy Bajo" seguido tenemos al aprendizaje colaborativo con porcentajes de 45,7\% en el grupo experimental con un nivel "Muy bajo" y 42,9\% en el grupo de control con nivel "Bajo", finalmente la gamificación la encontramos en porcentajes de 51,4\% en el grupo experimental y 42,9\% en el grupo control con nivel "Bajo", como se puede apreciar en la tabla 03.

Lo expuesto anteriormente, concuerda con el juicio realizado en la tesis doctoral de Morillas (2018), en el cual se verificó si la introducción de la gamificación y el trabajo colaborativo mejora el aprendizaje, frente a la no inclusión de la misma, por otra parte, en la tesis doctoral de Neyra (2020), se verificó a través de un pre test el nivel de inicio de los estudiantes antes de implementar las estrategias de ABP, para luego determinar el efecto de la aplicación de la estrategia sobre el aprendizaje significativo.

Como se puede apreciar, estos datos permitió identificar los niveles de uso de las estrategias metodológicas de la gamificación, aprendizaje colaborativo (AC) y aprendizaje basado en problemas, (ABP), donde se encontró que son situaciones recurrentes en diferentes contextos, el cual teóricamente tiene su fundamento en el postulado que, desde la pedagogía constructivista se necesita relacionar los contenidos y la experiencia, para alcanzar aprendizajes significativos, llegando a concluir que cuando los estudiantes no tienen este tipo de experiencias, no están en condiciones de alcanzar altos niveles de logro en las competencias académicas.

Considerando lo planteado en el segundo objetivo específico, identificar los elementos que debe contener un programa de capacitación sobre estrategias metodológicas para la enseñanza de la contabilidad se encontró al grupo experimental en un nivel "bajo" en el indicador "Hace uso del juego con herramientas virtuales" con un porcentaje del 65,7\% y el grupo de control obtuvo un porcentaje de $45,7 \%$, lo que quiere decir que entre los elementos que debe contener un programa en la estrategia de gamificación para la enseñanza de contabilidad se debe enfatizar en el uso de tecnología con diferentes 
aplicaciones, herramientas virtuales y juegos en red para la enseñanza de contabilidad, logrando mantener la motivación en el aula de clase, a su vez, en la estrategia metodológica aprendizaje colaborativo se encontró al grupo experimental en un nivel "bajo" en el indicador "Promueve a que los estudiantes se enseñen mutuamente a dominar temáticas contables y desarrollen competencias" con un porcentaje del $71,4 \%$ y el grupo de control obtuvo un 77,1\%, lo que refiere que se debe poner mayor atención en que el docente promueva el interaprendizaje entre compañeros y la estimulación del trabajo en equipo para la enseñanza de contabilidad.

Por otra parte, en la estrategia metodológica aprendizaje basado en problemas se encontró al grupo experimental en un nivel "muy bajo" en el indicador "El docente interactúa como un tutor para la resolución de problemas" con un porcentaje del 71,4\% y en el grupo de control el 68,6\%, lo que conlleva a poner mayor atención en que el docente apoye y asesore a la resolución de los problemas planteados en la clase y fomente la participación activa en todas las actividades planteadas para la resolución de problemas en la enseñanza de contabilidad, los hallazgos antes expuestos se pueden observar en las tablas 04, 05 y 06.

Como se observa, estos datos permitieron conocer lo que debe contener un programa con las estrategias de gamificación, aprendizaje colaborativo y aprendizaje basado en problemas, donde se encontró que los contenidos curriculares y el uso y aplicación de las estrategias son fundamentales en el proceso de enseñanza, por lo que, se concluye que, el educador en calidad de facilitador debe crear situaciones basada en las diferentes estrategias con el uso de herramientas adecuadas, incluyendo la interacción con juegos, motivando al trabajo en equipo y que se logre un aprendizaje dinámico en base a la experiencia, poniendo en práctica actividades que incentive al educando a la búsqueda de información y construcción de nuevos conocimientos, de manera individual y colectiva.

En relación a lo planteado en el tercer objetivo específico, el cual corresponde a determinar la percepción que tienen los estudiantes del grupo experimental y de control, de la enseñanza de contabilidad que reciben de sus docentes, antes de la implementación de nuevas estrategias en donde se obtuvo como resultado que la percepción de los estudiantes acerca de la enseñanza de contabilidad se encontró en nivel "mala" en ambos grupos, como se puede observar en la tabla 07 y figura 01 , con los porcentajes de $60 \%$ en el grupo experimental y el 57,1\% en el grupo de control, lo que se confirmó con la aplicación del estadístico U de Mann-Whitney el cual fue de 576,00 y el valor significativo (bilateral) de 0,672, lo que indica que tanto el grupo experimental y el de control antes de la aplicación de las estrategias metodológicas se encuentran en condiciones similares, lo cual permitió aceptar la hipótesis de investigación y rechazar la nula.

En consecuencia, se logró demostrar que antes del inicio de la experiencia los dos grupos participantes, uno como experimental y el otro como testigo se encontraron en situaciones similares, 
quienes no tienen una buena percepción respecto a la enseñanza de la contabilidad, lo que permitirá tener una medida objetiva de los cambios que se pueda generar luego de intervención.

Tomando en cuenta lo planteado en el cuarto objetivo específico establecer la percepción que tienen los estudiantes del grupo experimental y de control, de la enseñanza de contabilidad que reciben de sus docentes, después de la implementación de estrategias metodológicas, se pudo observar en los resultados que la percepción que tienen los estudiantes del grupo experimental y el grupo de control de la enseñanza de contabilidad que reciben de sus docentes, después de la implementación de estrategias metodológicas se ubicó en un porcentaje del 88,6\% en nivel muy bueno en el grupo experimental, mientras que el grupo de control se ubicó con un porcentaje de 62,9\% en nivel mala, como se observa en la tabla 10 y figura 02 .

Lo que antecedió, demuestra que la aplicación de las estrategias metodológicas influyó reflejando diferencias en ambos grupos, dicho resultado se confirmó con la aplicación del estadístico de prueba no paramétrica U de Mann-Whitney con un valor de 15,000 y el valor significativo (bilateral) de 0,00 lo que refiere que existe diferencia significativa entre ambos grupos.

De acuerdo a los resultados que se han venido realizando, se observa que dichos resultados coinciden con los hallazgos en la tesis doctoral realizada por Arrobo (2018) se establece una diferencia de medias de 2.08 puntos entre el pre test y post test de las variables en estudio, lo que demuestra que después de la aplicación de la estrategia metodológica se tiene diferencias significativas.

En efecto, se logró que después de la aplicación de las estrategias metodológicas se encuentren diferencias en los dos grupos participantes, los mismos que actuaron, uno como experimental y otro como testigo, lo que concluye, que la aplicación de las estrategias metodológicas influyó en el grupo experimental causando efectos significativos, permitiendo tener una medida objetiva en los cambios luego de la intervención.

En base a lo planteado en el quinto objetivo específico, Evaluar los efectos que tiene la aplicación del programa de estrategias metodológicas según la percepción de los estudiantes de la enseñanza de la contabilidad, en cada una de sus dimensiones, se obtuvo como resultado que con la aplicación de la estrategia metodológica de gamificación en el grupo experimental se obtuvo un nivel alto con un porcentaje de $85,7 \%$, por otro lado, con la aplicación de la estrategia metodológica aprendizaje colaborativo en el grupo experimental se obtuvo un nivel alto con un porcentaje del 80,0\%, asimismo, con la aplicación de la estrategia metodológica aprendizaje basado en problemas en el grupo experimental se obtuvo un nivel medio con un 65,7\%, como se observa en las tablas 12, 13 y 14.

Lo que refiere que la aplicación de cada una de las estrategias metodológicas causo un efecto positivo en los estudiantes, lo que se confirma en la contrastación de hipótesis a través de la aplicación del estadístico de prueba no paramétrica U de Mann-Whitney en la cual se observa los siguientes valores: 
43,000 en la gamificación, 31,000 en el aprendizaje colaborativo y el 61,000 en el aprendizaje basado en problemas, mientras que el valor significativo (bilateral) para las tres dimensiones es de 0,000 , lo que refiere que existen diferencias significativas con respecto a los efectos en la percepción de los estudiantes del grupo experimental sobre la enseñanza de contabilidad.

En vista de los resultados obtenidos a lo largo de los planteamientos realizados anteriormente, se puede observar que coincide con los resultados obtenidos en tesis doctoral realizada por Sánchez (2020), realizó un estudio cuasi experimental, en el cual se obtuvo como resultado que en el grupo experimental después de la aplicación de dicha estrategia logró un crecimiento a nivel medio con un porcentaje del $37,5 \%$, lo que genera una diferencia significativa y efecto positivo en los estudiantes, lo que permitió demostrar que innovar con la aplicación de la estrategia metodológica de gamificación, incide de manera positiva en el desempeño académico de los educando.

En lo que refiere a la estrategia metodológica de aprendizaje colaborativo, se encuentra que tiene coincidencia con los resultados obtenidos en la tesis doctoral realizada por Oblitas (2018), se obtuvo en los resultados que la aplicación que la estrategia metodológica de aprendizaje colaborativo demostró diferencia significativa en el grupo experimental, lo que indica que mejoró significativamente la capacidad de razonamiento lógico matemático en los educando lo que se comprobó con la aplicación del estadístico de prueba no paramétrica U de Mann- Whitney el mismo que refleja la significancia estadística p = 0,000.

Adicionalmente, en la tesis doctoral realizada por Cepeda (2017), se videncia que la aplicación de la estrategia metodológica aprendizaje colaborativo mejoran significativamente el proceso de enseñanza aprendizaje de los estudiantes, esto se confirma con la aplicación de la prueba Levene con un valor sig = $0,543>0,05$ con lo que se asume varianzas iguales y con una prueba $\mathrm{T}$ para la igualdad de medias para muestra independientes con un sig bilateral de 0,001 .

Como se puede contemplar, estos datos permitieron avaluar los efectos de la aplicación del programa estratégicas metodológicas de gamificación, aprendizaje colaborativo, aprendizaje basado en problemas, según la percepción de los estudiantes respecto a la enseñanza de la contabilidad, lo que concluye que, la aplicación de las estrategias antes mencionadas cauca un efecto positivo, sin embargo, en la estrategia metodológica aprendizaje basado en problemas, pese a que se aplicó al igual que las otras estrategias, está no causo mayor impacto en los estudiantes, pues se mantiene en un nivel medio, por lo que se asume que es una estrategia poco apropiada para la enseñanza de la asignatura de contabilidad.

Considerando lo planteado en el objetivo general, determinar el efecto del diseño y aplicación de estrategias metodológicas para mejorar la enseñanza de contabilidad desde la percepción de los estudiantes, encontramos que luego de haber aplicado el programa de estrategias metodológicas, se logra tener una mejoría en el enseñanza de contabilidad, según la percepción de los estudiantes en el grupo de estudio, donde la percepción de los estudiantes de la enseñanza de contabilidad en el pre test estuvo en 
nivel "Mala" con un 60,0\%, y cambio al 88,60\% a nivel "Muy buena", como se puede apreciar en la tabla 16 y figura 03 , lo que demuestra la eficacia del programa.

Lo expuesto anteriormente, se confirmó con la aplicación del estadístico U de Mann-Whitney obteniendo un valor significativo del 0,000 , lo que indica la existencia de diferencias significativas, quedando reflejado que después de la aplicación del programa de estrategias metodológicas los estudiantes mejoraron su percepción respecto a la enseñanza de contabilidad.

En efecto, se coincide con los resultados obtenidos en la tesis doctoral de Valdivieso (2019), considerada en los antecedes de esta investigación, se refleja diferencias de $Z=-6,401$ y $\mathrm{p}$ valor $=0,000$ lo que demuestra que las estrategias metodológicas utilizadas logran mejorar el conocimiento, convirtiéndose en la base para la elaboración de las planificaciones y así conseguir un mejor control en el proceso de enseñanza de cada asignatura, así mismo, Olivera (2020) en su artículo, concluyo que la contabilidad es una asignatura de campo con saberes específicos, por lo que es necesario desarrollar todas las estrategias metodológicas educativas para su abordaje y de esta manera lograr un aprendizaje significativo.

En tal sentido, estos datos permitieron determinar el efecto del diseño y aplicación de las estrategias metodológicas de gamificación, aprendizaje colaborativo y aprendizaje basado en problemas, en la mejora de la enseñanza de contabilidad según la percepción de los estudiantes, en la que se concluye, que la aplicación de dichas estrategias mejoran de manera significativa la enseñanza de contabilidad según la percepción de los estudiantes del grupo de estudio, lo que demuestra la eficacia del programa.

\section{PROPUESTA}

\section{PROGRAMA DE ESTRATEGIAS METODOLOGICAS PARA CONTABILIDAD}

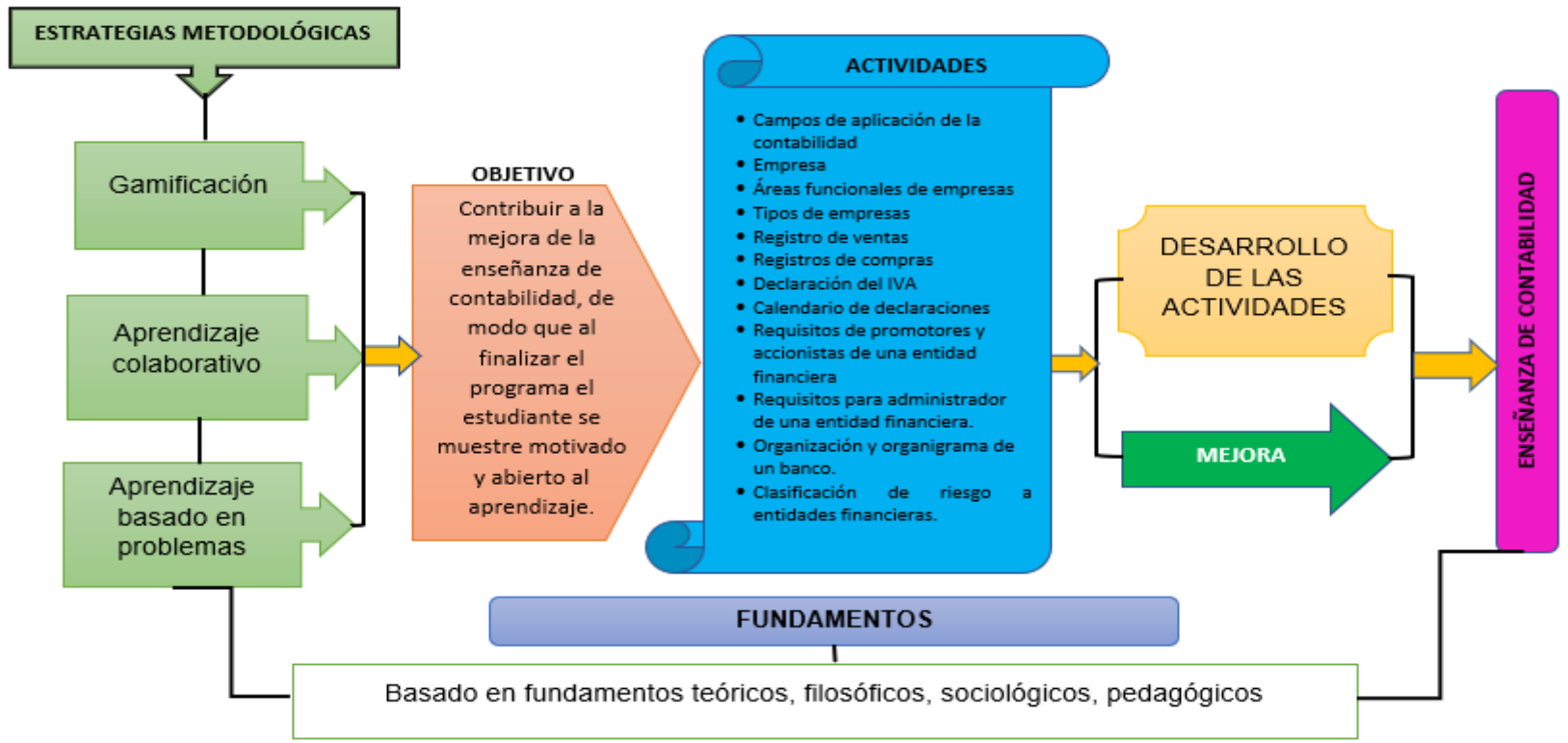

Fuente: Propuesta elaborada por la investiaadora. 
La propuesta de estrategias metodológicas denominada "Estrategias metodológicas para contabilidad, Mejorando mi conocimiento y enseñanza", está dirigido a dotar a los educadores que laboran en el área técnica contable e imparten las diferentes asignaturas de la figura profesional de contabilidad de métodos y estrategias pedagógicas que les permitan fortalecer el proceso de enseñanza.

El objetivo general de esta propuesta es contribuir a la mejora de la enseñanza de contabilidad, de modo que al finalizar el programa el estudiante se muestre motivado y abierto al aprendizaje, el cual se ejecutó en una institución educativa técnica.

La presente innovación educativa se la ha realizado sin fines de lucro, por lo tanto, no se esperó recuperación alguna de la inversión económica realizada, al contrario, se tuvo como finalidad contribuir con los resultados obtenidos en este experimento a cierta comunidad educativa en específico y que quizás pueda valer y ser de interés como un referente para la sociedad en general.

La propuesta de innovación educativa se planteó en un grupo de estudiantes de bachillerato de la figura profesional de contabilidad, quienes fueron denominados grupo experimental y que fueron expuesto a la experimentación de las estrategias metodológicas de gamificación, aprendizaje colaborativo y aprendizaje basado en problemas, durante la impartición de varias asignaturas de la figura profesional de contabilidad, con el propósito de mejorar el proceso de enseñanza en los docentes de contabilidad según la percepción de los estudiantes y lograr que estos se sientan motivados, abiertos al aprendizaje y sintiéndose capaces de afrontar problemas contables en el campo profesional en diferentes contextos y circunstancias que puedan surgir en la vida cotidiana. Esta propuesta consta de doce sesiones implementadas en las asignaturas de contabilidad general, paquetes contables y tributarios y contabilidad bancaria, dichas sesiones se desarrollaron en cuatro fases compuestas en: inicio, desarrollo, cierre y actividades. La modalidad de las sesiones se las realizó de manera virtual durante los meses de septiembre y octubre del año en curso (2021) con una duración de cuarenta minutos cada una.

\section{CONCLUSIONES}

Los estudiantes de los grupos experimental y control no tienen una percepción muy positiva respecto al uso de las estrategias de gamificación, trabajo colaborativo y aprendizaje basado en problemas, por parte de los docentes, quienes ven una escasa práctica de estas estrategias, tal como se puede observar en la Tabla $\mathrm{N}^{\circ} 3$.

Un programa con las estrategias de gamificación, aprendizaje colaborativo y aprendizaje basado en problemas debe contener la inclusión de juegos, motivación al trabajo en equipo, logros a través de un aprendizaje dinámico, basado en la experiencia, incentivando al estudiante a ser investigativo en la construcción de nuevos conocimientos de manera individual y colectiva. 
Antes de la aplicación de las estrategias metodológicas se encontraron situaciones similares en ambos grupos respecto a la percepción de la enseñanza de la contabilidad, la cual según la tabla $\mathrm{N}^{\circ} 07$ fue calificada como mala.

Después de la aplicación de las estrategias metodológicas se encontró un efecto positivo en el grupo experimental, respecto a la percepción de la enseñanza de la contabilidad, la cual según la tabla $\mathrm{N}^{\circ}$ 10 fue calificada como muy buena.

La aplicación de las estrategias metodologías tienen un efecto positivo en la enseñanza de contabilidad, aun así, pese a que la estrategia del aprendizaje basado en problemas se aplicó de igual manera que las otras estrategias, está no genero mayor impacto en los estudiantes, ya que obtuvo un nivel medio según se refleja en la tabla $\mathrm{N}^{\circ} 14$, por lo que se asume que es la menos apropiada para la enseñanza de la asignatura de contabilidad.

El diseño y aplicación de las estrategias metodologías de gamificación, aprendizaje colaborativo y aprendizaje basado en problemas, mejoran de manera significativa la enseñanza de contabilidad según la percepción de los estudiantes del grupo de estudio, lo que se refleja en la tabla $\mathrm{N}^{\circ} 16$, quedando demostrado la eficacia del programa. 


\section{REFERENCIAS}

Alan, N. D., \& Cortez, S. L. (2018). Procesos y Fundamentos de la Investigación Científica (Primera edición ed.). Editorial UTMACH. Obtenido de http://repositorio.utmachala.edu.ec/bitstream/48000/14232/1/Cap.4-

Investigaci\%C3\%B3n\%20cuantitativa\%20y\%20cualitativa.pdf

Alcaraz, A., Cruz, M., Guzman, M., Vidal, V., Pastor, M., Rodriguez, E., \& Sanchez, C. (2018). Didacticas de las ciencias sociales. Pearson Educación.

Arribas, E. J. (2017). La evaluación de los aprendizajes. Problemas y soluciones. Revista de Currículum y Formación de Profesorado, vol. 21, núm. 4. Obtenido de https://www.redalyc.org/pdf/567/56754639020.pdf

Arrobo Reyes, J. V. (2018). Método colaborativo y el aprendizaje de estudiantes en la asignatura de contabilidad básica de la carrera de mercadotecnia Universidad Laica Vicente. Obtenido de file://C:/Users/Personal/Downloads/Arrobo_rj\%20-\%20Resumen.pdf

Caamaño, S. C. (2018). Claves para potenciar la Atención/Concentración. CeACS Centro de Aprendizaje Campus Sur Universidad de Chile, 12.

Cuéllar, L. Z. (2020). Lineamientos para la enseñanza interdisciplinar en un programa de pregrado. Madrid.

Galeano, M. M. (2017). Diseño de Proyectos en la investigación. Fondo Medellín, Editorial Universidad EAFIT.

Gómez, M. (2017). Introducción a la Metodología de la Investigación. Edit. Brujas.

Hernández Ávila, C., \& Carpio, N. (2019). Introducción a los tipos de muestreo. ALERTA, $2 N^{\circ} 1,5$. Obtenido de https://alerta.salud.gob.sv/wp-content/uploads/2019/04/Revista-ALERTA-An\%CC\%83o2019-Vol.-2-N-1-vf-75-79.pdf

Jiménez Bonilla, E., \& Flores López, W. (2019). Consideraciones sobre el contenido curricular de matemáticas y su relación con las actitudes. Revista Electrónica De Conocimientos, Saberes Y Prácticas, 2(1), 36-46.ç. doi:https://doi.org/10.5377/recsp.v2i1.8165

MINISTRO DE EDUCACIÓN. (03 de Octubre de 2016). Acuerdos MINEDUC-ME-2016-00094-A. Obtenido de https://educacion.gob.ec/wpcontent/uploads/downloads/2017/01/guia_sugerencias_tareas_2016.pdf

Morillasm, C. (2018). Gamaficación de las aulas mediante las TIC: un cambio de paradigma en la enseñanza presencial frente a la docente tradicional Universidad Miguel Hernández. Obtenido de http://dspace.umh.es/bitstream/11000/3207/1/TD\%20\%20Morillas\%20Barrio,\%20C\%C3\%A9sar.pdf

Neyra, E. (2020). Aprendizaje basado en problemas para el aprendizaje significativo en matemática, en estudiantes de tercer año de secundaria, Chao 2019. Universidad Cesar Vallejo .

Oblitas, B. (2018). El Trabajo colaborativo y su influencia en el desarrollo de la capacidad de razonamiento lógico matemático en los estudiantes de primer grado de educación Secundaria de la IE: "Antonio Torres Araujo", Trujillo - 2017. Universidad Cesar Vallejo. Obtenido de https://alicia.concytec.gob.pe/vufind/Record/UCVV_72c0c559ce36f59f4c35b146f6432b2f 
Olivera, M. C. (mayo de 2020). La comprensión lectora en la enseñanza de la Contabilidad. Revista Experiencias del PCE, 4(4)., pp. 35-pp. 42. Obtenido de http://edicionesfhycs.fhycs.unam.edu.ar/index.php/experiencias

Páramo, B. P. (2018). La investigación en ciencias sociales : tecnicas de recolección de la información. Universidad Piloto de Colombia.

Rodríguez, J. A., \& Pérez, J. A. (2017). Métodos científicos de indagación y de construcción del conocimiento. Revista EAN, pp. 179 - 200. Obtenido de http://www.scielo.org.co/pdf/ean/n82/0120-8160ean-82-00179.pdf

Sellan, M. (2 de Enero de 2017). Importance of motivation in learning [Importancia de la motivación en el aprendizaje]. Sinergias educativa, 2(1), 1-5. doi:https://doi.org/10.37954/se.v2i1.20

Sánchez Pacheco, C. L. (2020). Gamificación personalizada para fortalecer aprendizajes significativos de la asignatura Matemática, en estudiantes de bachillerato de la ciudad de. Obtenido de https://repositorio.ucv.edu.pe/bitstream/handle/20.500.12692/54862/Sanchez_PCL-

SD.pdf?sequence $=1 \&$ isAllowed $=y$

Zimmermann, P. A., Stallings, L., Pierce, R. L., \& Largent, D. J. (2018). Classroom Interaction Redefined: Multidisciplinary Perspectives on Moving beyond Traditional Classroom Spaces to Promote Student Engagement [Redefinición de la interacción en el aula: perspectivas multidisciplinarias sobre cómo ir más allá de los espacios tradicionales del aula para promover la participación de los estudiantes]. Journal of Learning Spaces, v7 n1 p45-61. 